\title{
Integrated Characterization of Toxicity Distribution of Selected Heavy Metals in Stream Sediments Around Itakpe Iron Ore Mines, North Central Nigeria
}

\author{
Eneojo Godwin Ameh ${ }^{1}$, Sunday Ojochowu Idakwo ${ }^{1}$, Caroline Ojone $\mathrm{Ameh}^{2} \&$ Mercy Omojo Lekdukun $^{1}$ \\ ${ }^{1}$ Department of Earth Sciences, Faculty of Natural Sciences, Kogi State University, Anyigba, Kogi State, \\ Nigeria \\ ${ }^{2}$ Department of Geology, University of Port Harcourt, Rivers State, Nigeria \\ Correspondence: Eneojo Godwin Ameh, Department of Earth Sciences, Faculty of Natural Sciences, Kogi State \\ University, Anyigba, Kogi State, Nigeria. E-mail: ehiwill@gmail.com; ameh.eg@ksu.edu.ng
}

Received: February 20, 2016

Accepted: March 26, 2016

Online Published: December 27, 2016

doi:10.5539/esr.v6n1p109

URL: http://dx.doi.org/10.5539/esr.v6n1p109

\begin{abstract}
The results of the analysis were subjected to five (5) indices: contamination factor (CF); Ecological risk factor $\left(\mathrm{Er}^{\mathrm{i}}\right)$; Enrichment factor (EF); index of geo-accumulation (Igeo) and anthropogenic factor (AF). The CF results showed that the River PomPom dry and wet season sediments were collected in February and July respectively from $0 \mathrm{~cm}$ to $10 \mathrm{~cm}$ depth. Stainless steel spoon was used to collect the samples. The sediment samples were packaged, labeled and refrigerated for onward transportation to the laboratory. All samples were oven-dried $\left(50^{\circ} \mathrm{C}\right)$ for about 24 hours, sieved through -80 mesh. About $1.0 \mathrm{~g}$ of each sample was digested, filtered and the filtrates were ready for heavy metal analysis using Atomic sediments were most contaminated with $\mathrm{Fe}$ in both seasons, least contaminated with $\mathrm{Cd}$ at dry season and $\mathrm{Zn}$ in wet season. $\mathrm{The}^{\mathrm{E}} \mathrm{r}^{\mathrm{i}}$ factor revealed pollution hazard level of middle during wet season and tiny in dry season. $\mathrm{Pb}$ and $\mathrm{Ni}$ showed strong and middle hazard level during wet season and $\mathrm{Cu}$ revealed middle level during dry season. The enrichment factor indicated extremely enriched for $\mathrm{Fe}$ in all locations in both seasons while most heavy metals showed depletion to minimal enrichment. The Igeo for both seasons showed that Fe was moderately to highly polluted in all locations. During dry season, other heavy metals showed moderately to unpolluted while in wet season $\mathrm{Cd}, \mathrm{Ni}$ and $\mathrm{Pb}$ indicated moderately to very highly polluted. In both seasons, the $\% \mathrm{AF}$ was very high for $\mathrm{Fe}, \mathrm{Cu}, \mathrm{Pb}$. The $\mathrm{AF} \%$ was very high for $\mathrm{Ni}$ and $\mathrm{Cd}$ in wet season. All sites in both seasons have experienced deterioration but more in the wet season. From these indices, the stream sediments have been significantly contaminated with $\mathrm{Fe}, \mathrm{Ni}, \mathrm{Pb}$ and $\mathrm{Ni}$ in most locations in both seasons.
\end{abstract}

Keywords: Itakpe, ecological risk factor, contamination factor, index of geo-accumulation, anthropogenic factor

\section{Introduction}

In 1963, the Geological Survey of Nigeria (GSN) commenced exploratory work on Itakpe iron ore. The Itakpe iron ore deposit consist of two mine sites, one on the east and the other on the west. Work on the western mine has since stopped. Conspicuous on the eastern mine are: view point, overburden dumps, primary and secondary crushers, washing pond, concentrate area and tailing dam point. The area has rough topography. Sandwiched within this mine is River PomPom.

Heavy metals are serious pollutants in natural environment due to their toxicity, persistence and bioaccumulation problems (Seshan et al., 2010). The impact of anthropogenic activities is most often felt by streams adjacent to mining activities. Heavy metals are rapidly removed from water bodies and deposited onto the sediments. Sediment analysis offers advantages over water analysis for the control and detection of metal pollution in sediments (Seshan et al., 2010). Surface sediments also exchange with suspended materials, thereby affecting the release of metals to the overlying water. Therefore, the top few centimeters of the sediments reflect the continuously changing present day degree of contamination, whereas the bottom sediments record its history (Periera et al., 2008). Metals are introduced into stream sediments from mining activities, run-off, atmospheric deposition, as well as upstream run-off accumulation into the sediments.

Little information, however, exist on the impact of these mining activities on the environmental loading of heavy 
metals and particularly the extent to which sediments have been contaminated by metal-rich iron-ore mining activities (Periera et al., 2008).

The main objective of the study therefore, is to examine the metal concentrations in top sediments and evaluate the extent of heavy metal contamination arising from iron-ore mining related activities. This is because sediments are sensitive indicators for monitoring contaminants in aquatic environments. This study was carried out over the period of twelve months (12 months) in two phases: dry and wet seasons. All analyses were carried out in the Faculty of Agriculture Laboratory, Kogi State University, Nigeria.

\section{Geology of Itakpe}

The study area is on Kabba topographical sheet 246, southeast Nigeria (Figure 1). It falls within the geographical location of Longitude $6^{\circ} 15^{\prime}$ to $6^{\circ} 30^{\prime} \mathrm{E}$ and Latitude $7^{\circ} 30^{\prime}$ to $7^{\circ} 45^{\prime} \mathrm{N}$. Specifically, the study area lies between Longitude $6^{\circ} 17^{\prime}$ to $6^{\circ} 20^{\prime} \mathrm{E}$ and Latitude $7^{\circ} 36^{\prime}$ to $7^{\circ} 37^{\prime} 30^{\prime \prime} \mathrm{N}$. The Itakpe iron ore deposit is localized within the gneiss- migmatite-quartzite unit of the Nigerian basement complex (Olade, 1978). These basement rocks are overlain by a sequence of low-grade metamorphic rocks and intruded by a suite of charnockitic and granitoid rocks. The rocks exposed on the Itakpe ridge are gneisses, quartzites, amphibolites, schists, charnockites, granites and pegmatites (Figure 1).

Gneisses: are most widespread rocks at Itakpe and occur as layers of about 50m thick, alternating with bands and lenses of ferruginous quartzites. The principal variety is the strongly foliated banded gneiss characterized by alternating leucocratic (quartz feldspar) and melanocratic (biotite-hornblende) bands. A homogenous, non foliated variety (granitic gneiss) occurs where bodies of quartz and feldspar are distributed or impregnated within a matrix of mafic minerals. Four gneises were recognized: biotite gneiss and garnet-biotite gneiss (occur in the western part), hornblende-biotite gneiss and pyroxene-hornblende gneiss. The latter two are confined to the central and eastern parts of the ridge (Olade, 1978).

Quartzites: are dominantly ferruginous and form the main rock type on the ridge. They occur as bands and lenses of about 10-60 m wide, in alternation with gneisses. Non- ferruginous or pure quartzites are rare, although it outcrops in adjacent ridges. Three structural-textural varieties of quartzites were recognized: massive, banded and metamorphosed. In the schistose variety, both the quartz and iron oxides are platy and commonly reach lengths $>$ 5mm (Olade, 1978).

Amphibolites and Schists: These rocks occur in subsurface as lenses and sheets that range in width from $<1$ to $25 \mathrm{~m}$. They showed sharp contacts with the surrounding country rocks. The amphibolites were medium-grained and exhibit weak schistocity, which results from the alignment of hornblende and biotite plates. The biotite schists were less abundant and about $20 \mathrm{~m}$ in width. They are medium to coarse grained, dominantly brownish biotite, minor hornblende, quartz and iron oxide. The biotite schist shows close proximity with the ferruginous quartzite. Sometimes it shows sharp contacts with the ore and in such cases the schist is accompanied by increased iron oxide content (Olade, 1978).

Charnockites and Granites: Charnockite body intrudes both the gneisses and ferruginous quartzites along the northern flank of the Itakpe ridge. The rock is fine to medium grained in texture. It is composed dominantly of felsic minerals with minor interstitial grains of orthopyroxene and iron oxides.

Other granitic bodies occur as dykes and sills, which exhibit sharp contacts with the gneisses. They are non-porphyritic and range in composition from hornblende- biotite granite to quartz diorite (Olade, 1978).

\section{Materials and Methods}

Stream sediments were collected both at dry and wet seasons within the period of twelve (12) months (Figure 2). Surface sediment samples were collected between $0 \mathrm{~cm}$ to $10 \mathrm{~cm}$ depth beneath the aqueous layer. The locations of the samples were recorded with a global positioning system (GPS). Plant debris, stones were removed and sample clumps disaggregated. The samples were air-dried for 48 hours till the moisture was removed. The samples were crushed to pass through a $2-\mathrm{mm}$ mesh, grounded and sieved to pass through $<60 \mu \mathrm{m}$. The clay fractions were preserved in plastic bags to avoid loss of fines and kept in a dryer in order to determine the heavy metal content. A control point of about 500m away with similar geology but no iron-ore deposit was chosen. Sediment sample weight of 1.0grams each was digested using a multi-acid digestion (near total digestion) of $\mathrm{HNO}_{3}-\mathrm{HCl}-\mathrm{HClO}_{4}-\mathrm{HF}$ combinations in a triangular flask, slaked in slacker and the volume determined. All apparatus were soaked overnight using $20 \%$ hydrogen nitrate and rinsed with distilled water three times. The heavy metal test was carried out in the AAS heavy metal room of the Faculty of Agriculture, Kogi State University, Anyigba, Nigeria. 


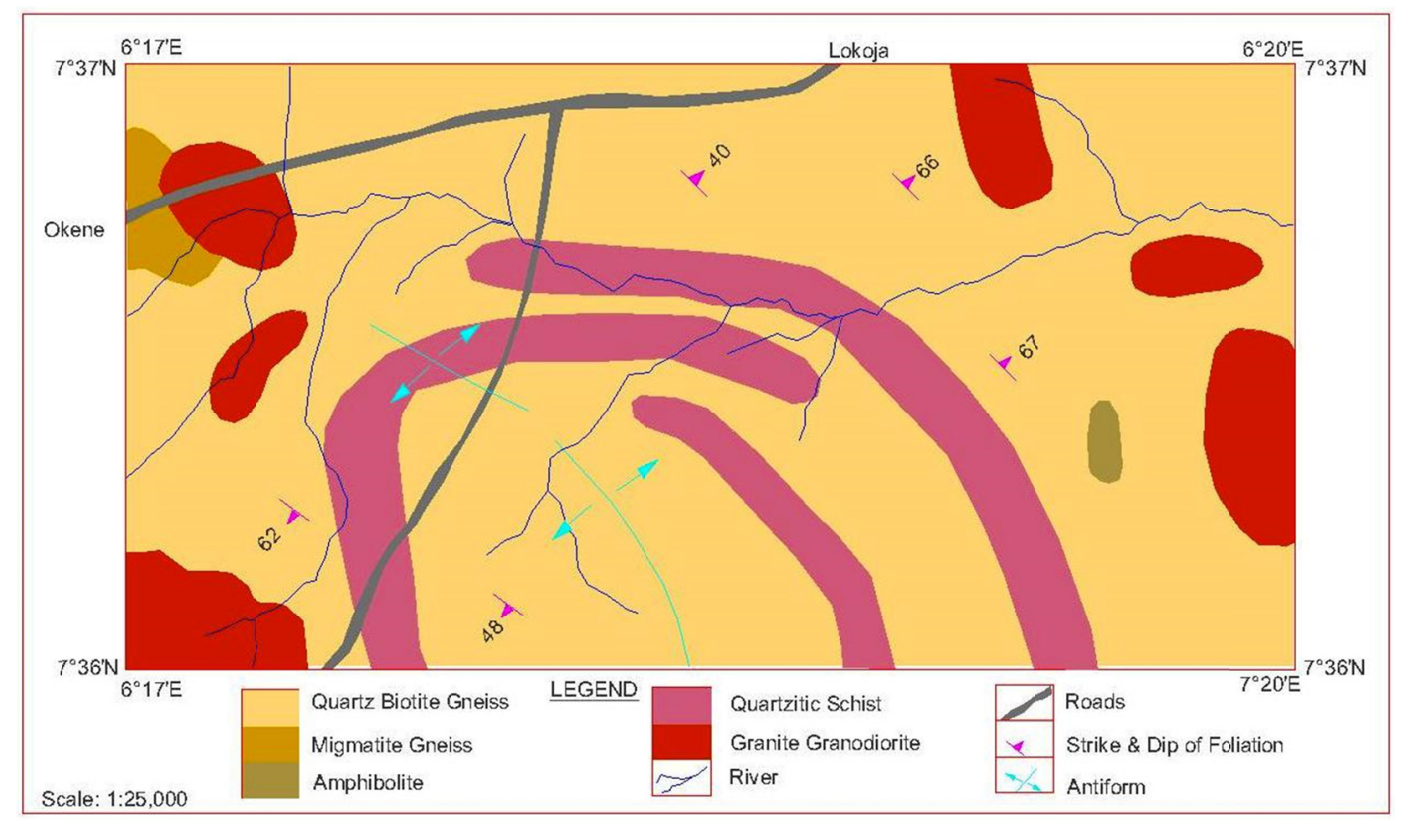

Figure 1. Geological map of Itakpe (Modified after Odigi, 2002)

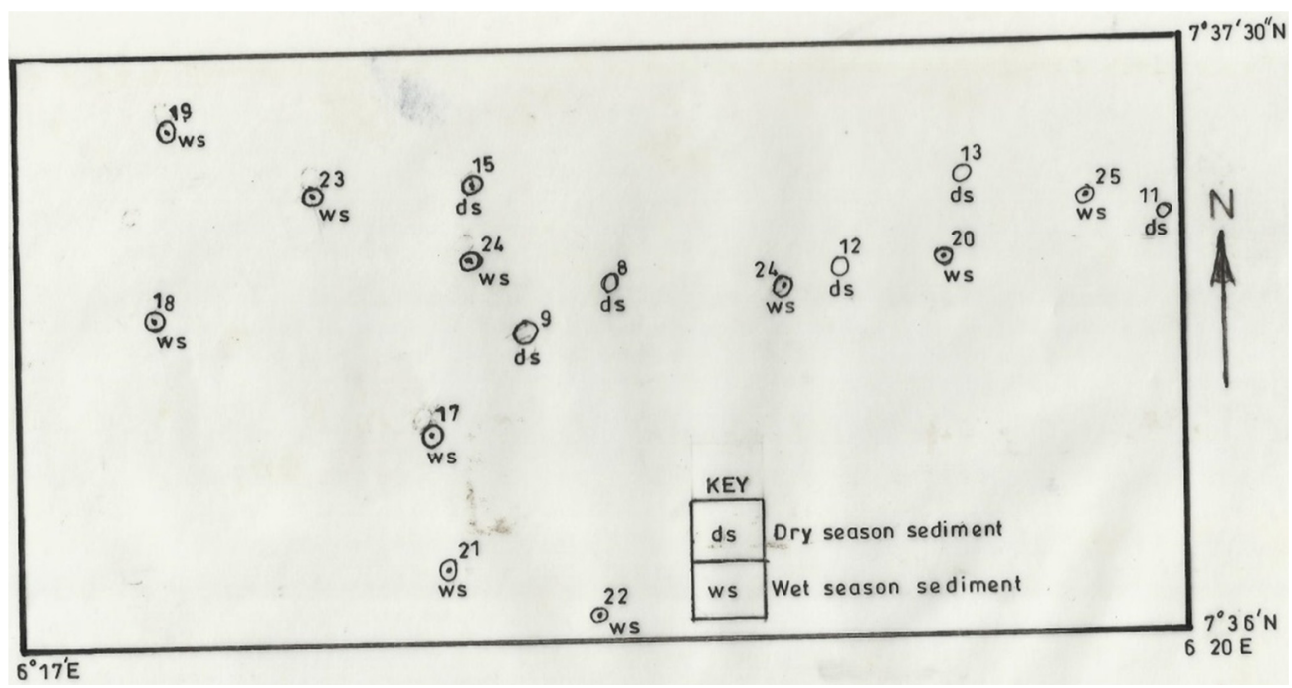

Figure 2. Sample location points of the study area

\section{Quantification Indices}

(i) Contamination factor (CF): This was suggested by Hakanson (1980) as follows: $C_{f}^{i}=C_{i} C_{i r}$, where $C_{i}$ is content of metal; $\mathrm{C}_{\mathrm{ri}}$ is the reference value (control value in this study) of metal $\mathrm{i}$. The following terminologies were used to describe the contamination factor: $C_{f}^{i}<1$ low contamination factor; $1<=C_{f}^{i}<3$, moderate contamination factor; $3<=\mathrm{C}_{\mathrm{f}}^{\mathrm{i}}<6$, considerable contamination factor; and $\mathrm{C}_{\mathrm{f}}^{\mathrm{i}}>=6$, very high contamination factor.

(ii) Ecological risk factor: An ecological risk factor $\left(\mathrm{Er}^{\mathrm{i}}\right)$ was used to quantitatively expressed the potential ecological risk of a given contaminant as suggested by Hakanson, (1980) as follows: $\operatorname{Er}^{\mathrm{i}}=\operatorname{Tr}^{\mathrm{i}} \times \mathrm{C}_{\mathrm{f}}^{\mathrm{i}}$, where $\operatorname{Tr}^{\mathrm{i}}$ is the toxic-response factor for a given substance and $\mathrm{C}_{\mathrm{f}}^{\mathrm{i}}$ is the contamination factor. The $\operatorname{Tr}^{\mathrm{i}}$ values of heavy metals by Hakanson (1980) are given below (Table 1): The following terminologies were used to describe the risk factor: $\operatorname{Er}^{\mathrm{i}}<40$, low potential ecological risk; $40<=\operatorname{Er}^{\mathrm{i}}<80$, moderate potential ecological risk; $80<=\operatorname{Er}^{\mathrm{i}}<$ 160 , considerable potential ecological risk; $160<=\operatorname{Er}^{\mathrm{i}}<320$, high potential ecological risk; and $\mathrm{Er}^{\mathrm{i}}>=320$, very high ecological risk. 
Table 1. Toxicity factor by Hakanson (1980)

\begin{tabular}{llllll}
\hline Elements & $\mathrm{Cd}$ & $\mathrm{Cu}$ & $\mathrm{Pb}$ & $\mathrm{Ni}$ & $\mathrm{Zn}$ \\
\hline Toxic reference factor & 30 & 5 & 5 & 3 & 1 \\
\hline
\end{tabular}

(iii) Enrichment factor (EF): The formula for computing EF is $=\left(C_{i} / C_{i e}\right)_{s} /\left(C_{i} / C_{i e}\right)_{R S}$, where $C_{i}$ is thecontent of element (i) in the sample of interest or selected reference sample, and $\mathrm{C}_{\mathrm{ie}}$ is the content of immobile element in the sample or the sample of interest, and $\left(\mathrm{C}_{\mathrm{i}} / \mathrm{C}_{\mathrm{ie}}\right)_{\mathrm{RS}}$ is the heavy metal to immobile element ratio in the selected reference sample. The selected reference sample is usually an average crust or local background sample. In this study, the reference sample is the control value. The immobile element is often taken to be $\mathrm{Li}, \mathrm{Sc}, \mathrm{Zr}$ and $\mathrm{Ti}$. Sometimes Fe or Mn has been used (Qingjie et al., 2008). Fe was used as the immobile element in this study. According to Sutherland (2000), five contamination categories are generally recognized on the basis of the enrichment factor: $\mathrm{EF}<2$, depletion to minimal enrichment; $2<=\mathrm{EF}<5$, moderate enrichment; $5<=\mathrm{EF}<20$, significant enrichment; $20<=\mathrm{EF}<40$, very high enrichment; and $\mathrm{EF}>40$, extremely high enrichment.

(iv) Index of geo-accumulation (Igeo): This was defined by Muller, (1969) in order to determine heavy metal concentration in sediments by comparing current concentrations with control values. It is calculated by the following equation: Igeo $=\log _{2}\left[\mathrm{C}_{\mathrm{i}} /\left(1.5 \mathrm{C}_{\mathrm{ri}}\right)\right]$ where $\mathrm{C}_{\mathrm{i}}$ is the measured concentration of heavy metal (i) in the sediment, and $\mathrm{C}_{\mathrm{ri}}$ is the geochemical background concentration or reference value forthe given metal in the environment. The Igeo is classified into seven classes: Igeo $<0$, class 0 , unpolluted; $0<$ Igeo $<=1$, class 1 , from unpolluted to moderately polluted; $1<$ Igeo $<=2$, class 2 , moderately polluted; $2<$ Igeo $<=3$, class 3 , from moderately to strongly polluted; $3<$ Igeo $<=4$, class 4 , strongly polluted; $4<$ Igeo $<=5$, class 5 , from strongly to extremely polluted; and Igeo $>5$, class 6 , extremely polluted.

(v)Anthropogenic factor (AF): This was calculated for the top sediment samples. The $A F=C_{s} / C_{c}$, where $C_{s}=$ concentration of heavy metal in sediments; $\mathrm{C}_{\mathrm{c}}=$ concentration of heavy metals in control values. The result would indicate the extent of anthropogenic influence on heavy metals in top sediment samples.

\section{Results}

Tables 2. Itakpe dry and wet seasons sediment (mg/L) and descriptive statistics

\begin{tabular}{|c|c|c|c|c|c|c|c|c|c|c|}
\hline & $\mathrm{Na}$ & $\mathrm{K}$ & $\mathrm{Ca}$ & $\mathrm{Mg}$ & $\mathrm{Fe}$ & $\mathrm{Cu}$ & $\mathrm{Zn}$ & $\mathrm{Pb}$ & $\mathrm{Ni}$ & $\mathrm{Cd}$ \\
\hline Min. & 14.51 & 58.81 & 27.35 & 3.08 & 9360.51 & 0.03 & 0.46 & 0.01 & 0.26 & 0.38 \\
\hline Max & 23.45 & 181.07 & 62.41 & 8.49 & 35525.00 & 2.17 & 1.86 & 0.45 & 1.46 & \\
\hline Mean & 17.80 & 135.71 & 44.76 & 5.14 & 20714.52 & 0.80 & 1.06 & 0.15 & 0.82 & 1.05 \\
\hline Std Error & 1.41 & 21.58 & 6.01 & 1.03 & 4590.16 & 4.92 & 11.94 & 7.15 & 15.62 & 8.16 \\
\hline $\mathrm{T}-$ test & 12.60 & 6.00 & 7.44 & 5.01 & 4.51 & 1.77 & 2.34 & 2.10 & 2.63 & 1.75 \\
\hline Average $\mathrm{Cv}$ value & 20.41 & 107.40 & 16.79 & 5.68 & 755.00 & 0.06 & 1.20 & 0.07 & 0.75 & 1.70 \\
\hline Std. Dev & 3.46 & 43.59 & 14.73 & 2.51 & 11243.47 & 1.06 & 0.59 & 0.18 & 0.42 & 0.56 \\
\hline & $\mathrm{Na}$ & $\mathrm{K}$ & $\mathrm{Ca}$ & $\mathrm{Mg}$ & $\mathrm{Fe}$ & $\mathrm{Cu}$ & $\mathrm{Zn}$ & $\mathrm{Pb}$ & $\mathrm{Ni}$ & $\mathrm{Cd}$ \\
\hline Min & 7.24 & 38.61 & 26.15 & 2.09 & 8474.24 & 0.26 & 0.10 & 0.04 & 0.01 & 0.35 \\
\hline Max & 18.76 & 102.23 & 114.25 & 5.44 & 45250.00 & 1.71 & 1.94 & 1.37 & 1.84 & 1.59 \\
\hline Mean & 13.80 & 70.57 & 68.16 & 3.66 & 22191.25 & 1.07 & 0.90 & 0.44 & 0.52 & 0.96 \\
\hline Std Error & 1.53 & 7.47 & 10.26 & 0.38 & 5190.94 & 6.05 & 13.99 & 9.54 & 11.36 & 9.66 \\
\hline $\mathrm{T}-$ test & 8.27 & 9.44 & 6.64 & 9.53 & 4.01 & 2.16 & 3.12 & 1.82 & 2.77 & 2.55 \\
\hline Average $\mathrm{Cv}$ value & 107.04 & 36.29 & 28.25 & 3.55 & 562.40 & 0.39 & 1.73 & 0.02 & 0.03 & 0.06 \\
\hline Std. Dev & 3.75 & 22.42 & 30.78 & 1.16 & 14120.43 & 0.57 & 0.52 & 0.53 & 0.59 & 0.44 \\
\hline
\end{tabular}


Table 3a. Correlation matrix of dry season sediments

\begin{tabular}{|c|c|c|c|c|c|c|c|c|c|c|}
\hline & $\mathrm{Na}$ & K & $\mathrm{Ca}$ & $\mathrm{Mg}$ & $\mathrm{Fe}$ & $\mathrm{Cu}$ & $\mathrm{Zn}$ & $\mathrm{Pb}$ & $\mathrm{Ni}$ & $\mathrm{Cd}$ \\
\hline $\mathrm{Na}$ & 1 & .747 & $.953^{* *}$ & $.945^{* * *}$ & .389 & -.524 & -.485 & $.878^{*}$ & -.561 & -.691 \\
\hline K & & 1 & .756 & .705 & -.291 & $-.954^{* *}$ & .075 & .431 & -.357 & -.788 \\
\hline $\mathrm{Ca}$ & & & 1 & $.933^{* *}$ & .312 & -.547 & -.426 & .758 & -.457 & -.793 \\
\hline $\mathrm{Mg}$ & & & & 1 & .457 & -.469 & -.635 & $.896^{*}$ & -.726 & -.591 \\
\hline $\mathrm{Fe}$ & & & & & 1 & .559 & $-.920^{* *}$ & .718 & -.497 & .173 \\
\hline $\mathrm{Cu}$ & & & & & & 1 & -.337 & -.160 & .186 & .705 \\
\hline $\mathrm{Zn}$ & & & & & & & 1 & -.799 & .772 & -.154 \\
\hline $\mathrm{Pb}$ & & & & & & & & 1 & -.772 & -.307 \\
\hline $\mathrm{Ni}$ & & & & & & & & & 1 & -.082 \\
\hline$\underline{\mathrm{Cd}}$ & & & & & & & & & & \\
\hline
\end{tabular}

** Correlation is significant at the 0.01 level.* Correlation is significant at the 0.05 level.

Table 3b. Correlation matrix of rainy season sediments

\begin{tabular}{|c|c|c|c|c|c|c|c|c|c|c|}
\hline & $\mathrm{Na}$ & K & $\mathrm{Ca}$ & $\mathrm{Mg}$ & $\mathrm{Fe}$ & $\mathrm{Cu}$ & $\mathrm{Zn}$ & $\mathrm{Pb}$ & $\mathrm{Ni}$ & $\mathrm{Cd}$ \\
\hline $\mathrm{Na}$ & 1 & -.052 & -.092 & .480 & -.235 & -.004 & .167 & .481 & $.674^{*}$ & .051 \\
\hline K & & 1 & $.736^{*}$ & $.686^{*}$ & $.777^{*}$ & $-.763^{*}$ & .263 & -.033 & -.157 & -.381 \\
\hline $\mathrm{Ca}$ & & & 1 & .530 & .457 & -.529 & .108 & -.154 & -.023 & -.635 \\
\hline $\mathrm{Mg}$ & & & & 1 & .335 & $-.668^{*}$ & .150 & .255 & .367 & -.420 \\
\hline $\mathrm{Fe}$ & & & & & 1 & -.528 & .523 & .349 & -.340 & -.249 \\
\hline $\mathrm{Cu}$ & & & & & & 1 & .240 & -.110 & -.334 & $.728^{*}$ \\
\hline $\mathrm{Zn}$ & & & & & & & 1 & .391 & -.357 & .336 \\
\hline $\mathrm{Pb}$ & & & & & & & & 1 & .425 & -.192 \\
\hline $\mathrm{Ni}$ & & & & & & & & & 1 & -.535 \\
\hline $\mathrm{Cd}$ & & & & & & & & & & 1 \\
\hline
\end{tabular}

*Correlation is significant at the 0.05 level.

Table 4. Anthropogenic factor of dry and wet season sediments

\begin{tabular}{|c|c|c|c|c|c|c|c|c|}
\hline & & Dry & & & Wet & & & \\
\hline $\begin{array}{l}\text { Heavy } \\
\text { metals } \\
(\mathrm{mg} / \mathrm{l})\end{array}$ & $\begin{array}{l}\text { Mean } \\
\text { measured } \\
\text { concentration }\end{array}$ & $\begin{array}{l}\text { Control point } \\
\text { concentration }\end{array}$ & $\begin{array}{c}\text { Anthropogenic } \\
\text { factor (AF) }\end{array}$ & $\% \mathrm{AF}$ & $\begin{array}{l}\text { Mean } \\
\text { measured } \\
\text { concentration }\end{array}$ & $\begin{array}{l}\text { Control point } \\
\text { concentration }\end{array}$ & $\begin{array}{l}\text { Anthropoge } \\
\text { nic factor }\end{array}$ & $\% \mathrm{AF}$ \\
\hline $\mathrm{Fe}$ & 20714.52 & 755.00 & 27.44 & 96.48 & 22191.25 & 562.4 & 39.46 & 97.53 \\
\hline $\mathrm{Cu}$ & 0.7983 & 0.06 & 13.31 & 93.01 & 1.07 & 0.39 & 2.74 & 73.29 \\
\hline $\mathrm{Zn}$ & 1.06 & 1.20 & 0.88 & 46.90 & 0.9 & 1.73 & 0.52 & 34.22 \\
\hline $\mathrm{Pb}$ & 0.15 & 0.07 & 2.14 & 68.18 & 0.44 & 0.2 & 2.2 & 68.75 \\
\hline $\mathrm{Ni}$ & 0.8183 & 0.75 & 1.09 & 52.18 & 0.52 & 0.03 & 17.33 & 94.55 \\
\hline $\mathrm{Cd}$ & 1.0533 & 1.70 & 0.62 & 38.26 & 0.96 & 0.06 & 16 & 94.12 \\
\hline
\end{tabular}

$\mathrm{AF}=\mathrm{C}_{\mathrm{s}} / \mathrm{C}_{\mathrm{v}} \mathrm{C}_{\mathrm{s}}=$ measured concentration; $\mathrm{C}_{\mathrm{v}}=$ control concentration.

Table 5a. The $\mathrm{I}_{\mathrm{geo}}$ of heavy metals in dry season sediments

\begin{tabular}{lllllll}
\hline \multicolumn{7}{c}{ Heavy metalsSample Locations } \\
& ITK08 & ITK09 & ITK11 & ITK12 & ITK13 & ITK15 \\
\hline $\mathrm{Fe}$ & 4.97 & 3.25 & 4.44 & 4.78 & 3.51 & 3.05 \\
$\mathrm{Cu}$ & 1.87 & -1.59 & 4.57 & 4.59 & -0.36 & -0.85 \\
$\mathrm{Zn}$ & -0.43 & -1.63 & -0.23 & 0.05 & -1.61 & -1.97 \\
$\mathrm{~Pb}$ & 0.31 & -3.39 & 1.31 & 2.87 & -1.81 & -2.39 \\
$\mathrm{Ni}$ & -1.23 & -0.44 & -0.16 & 0.38 & -0.37 & -2.11 \\
$\mathrm{Cd}$ & -2.60 & -1.24 & -0.73 & -0.55 & -1.14 & -2.75 \\
\hline
\end{tabular}

$\mathrm{I}_{\text {geo }}=\log 2\left[\left(\mathrm{C}_{\mathrm{i}}\right) /(1.5 * \mathrm{Cv})\right]$ : Where $\mathrm{C}_{\mathrm{i}}=$ measured concentration; $\mathrm{Cv}=$ control concentration; $1.5=$ a factor for possible variations in reference concentration due to lithologic differences. 
Table 5b. Geo-accumulation of heavy metal classes (Wenjin and Xinqing, 2013)

\begin{tabular}{llllllll}
\hline Degree & 0 & 1 & 2 & 3 & 4 & 5 & 6 \\
\hline $\begin{array}{l}\mathrm{I}_{\text {geo }} \\
\text { Polluti } \\
\text { on }\end{array}$ & $<0$ & {$[0,1]$} & {$[1,2]$} & {$[2,3]$} & {$[3,4]$} & {$[4,5]$} & {$[5,6]$} \\
Degree & $\begin{array}{l}\text { No } \\
\text { pollution }\end{array}$ & $\begin{array}{l}\text { Light } \\
\text { pollution }\end{array}$ & $\begin{array}{l}\text { Partial } \\
\text { pollution }\end{array}$ & $\begin{array}{l}\text { Middle } \\
\text { pollution }\end{array}$ & $\begin{array}{l}\text { Heavier } \\
\text { pollution }\end{array}$ & $\begin{array}{l}\text { Heavy } \\
\text { pollution }\end{array}$ & $\begin{array}{l}\text { Serious } \\
\text { polluti } \\
\text { on }\end{array}$ \\
\hline
\end{tabular}

Table 6. Igeo of heavy metals in Itakpe wet season sediments

\begin{tabular}{lllllll}
\hline Sample location & \multicolumn{6}{l}{ Heavy metals $(\mathrm{mg} / \mathrm{l})$} \\
\hline & $\mathrm{Fe}$ & $\mathrm{Cu}$ & $\mathrm{Zn}$ & $\mathrm{Pb}$ & $\mathrm{Ni}$ & $\mathrm{Cd}$ \\
ITK17 & 5.75 & 1.40 & -1.39 & -1.74 & -2.18 & 3.84 \\
ITK18 & 5.22 & 0.93 & -0.42 & -1.89 & 1.00 & 3.54 \\
ITK19 & 5.34 & 0.95 & -2.06 & -1.89 & 5.35 & 2.78 \\
ITK20 & 3.60 & -0.34 & -1.42 & -2.12 & -2.18 & 2.90 \\
ITK21 & 4.01 & 1.55 & -1.09 & 2.19 & 4.12 & 4.15 \\
ITK22 & 3.87 & 1.23 & -1.29 & 1.85 & 3.94 & 4.04 \\
ITK23 & 5.38 & 1.48 & -1.69 & 1.60 & 4.08 & 3.58 \\
ITK24 & 3.33 & -1.18 & -4.68 & -2.94 & 1.15 & 1.96 \\
ITK25 & 3.46 & -0.67 & -2.64 & -0.20 & 3.04 & 2.50 \\
\hline
\end{tabular}

Table 7. Grading standard of $\mathrm{Ei}_{\mathrm{r}}$ and RI (Hakanson, 1980)

\begin{tabular}{lll}
\hline Pollution coefficient $\mathrm{E}_{\mathrm{r}}^{\mathrm{i}}$ & Pollution index RI & Pollution level (ecological hazard) \\
\hline$\leq 40$ & $\leq 150$ & Tiny \\
$40-79$ & $150-299$ & Middle \\
$80-159$ & $300-600$ & Strong \\
$160-320$ & $>600$ & Very strong \\
$>320$ & - & Serious \\
\hline
\end{tabular}

Table 8. Assessment of potential ecological risk of dry season heavy metals in sediment

\begin{tabular}{llllll}
\hline Elements & Toxicity coefficient & Measured value $(\mathrm{mg} / \mathrm{l})$ & Ratio of $\mathrm{C}_{\mathrm{s}} / \mathrm{C}_{\mathrm{v}}(\mathrm{mg} / \mathrm{l})$ & $\mathrm{E}_{\mathrm{r}}^{\mathrm{i}}$ & Hazard level \\
\hline $\mathrm{Cd}$ & 30 & 1.05 & 0.62 & 18.6 & Tiny \\
$\mathrm{Cu}$ & 5 & 0.80 & 13.33 & 66.65 & Middle \\
$\mathrm{Pb}$ & 5 & 0.15 & 2.14 & 10.70 & Tiny \\
$\mathrm{Ni}$ & 3 & 0.82 & 1.09 & 3.27 & None \\
$\mathrm{Zn}$ & 1 & 1.06 & 0.88 & 0.88 & None \\
$\mathrm{RI}$ & & & 100.1 & Tiny \\
\hline
\end{tabular}

Table 9. Assessment of potential ecological risk of heavy metals in wet season sediment

\begin{tabular}{llllll}
\hline Elements & Toxicity coefficient & Measured value $(\mathrm{mg} / \mathrm{l})$ & Ratio of $\mathrm{C}_{\mathrm{s}} / \mathrm{C}_{\mathrm{v}}(\mathrm{mg} / \mathrm{l})$ & $\mathrm{E}_{\mathrm{r}}^{\mathrm{i}}$ & Hazard level \\
\hline $\mathrm{Cd}$ & 30 & 0.96 & 0.063 & 1.89 & None \\
$\mathrm{Cu}$ & 5 & 1.07 & 2.74 & 13.7 & Tiny \\
$\mathrm{Pb}$ & 5 & 0.44 & 22 & 110 & Strong \\
$\mathrm{Ni}$ & 3 & 0.52 & 17.33 & 51.99 & Middle \\
$\mathrm{Zn}$ & 1 & 0.90 & 0.52 & 0.52 & None \\
$\mathrm{RI}$ & & & 178 & Middle \\
\hline
\end{tabular}


Table 10a. Enrichment factor (EF) of heavy metals in dry season sediments

\begin{tabular}{lllllll}
\hline Heavy & \multicolumn{7}{c}{ Sample locations } \\
\hline Metals (mg/l) & ITK08 & ITK09 & ITK11 & ITK12 & ITK13 & ITK15 \\
$\mathrm{Fe} / 100$ & 0.4214 & 0.295 & 0.255 & 0.267 & 0.349 & 0.32 \\
$\mathrm{Cu}$ & 0.12 & 0.04 & 1.1 & 0.88 & 0.07 & 0.07 \\
$\mathrm{Zn}$ & 0.02 & 0.03 & 0.04 & 0.04 & 0.03 & 0.03 \\
$\mathrm{~Pb}$ & 0.04 & 0.01 & 0.12 & 0.16 & 0.03 & 0.02 \\
$\mathrm{Ni}$ & 0.01 & 0.08 & 0.04 & 0.05 & 0.07 & 0.03 \\
$\mathrm{Cd}$ & 0 & 0.04 & 0.03 & 0.02 & 0.04 & 0.02 \\
\hline
\end{tabular}

Table 10b. Enrichment factor of heavy metal classes (After Wenjie and Xinqing, 2013)

\begin{tabular}{llllllll}
\hline EF & $<0.25$ & $0.25-0.5$ & $0.5-0.75$ & $0.75-1.5$ & $1.5-2$ & $2-4$ & $>4$ \\
\hline Enrichment & extremely & strong & weak & proximity & weak & strong & Extremely \\
Degree & depleted & depletion & depletion & enrichment & enrichment & $\begin{array}{l}\text { enrichment } \\
\text { enrichment }\end{array}$ \\
\hline
\end{tabular}

Table 11. Enrichment factor (EF) of Itakpe wet season sediments

\begin{tabular}{lllllll}
\hline Sample location & \multicolumn{6}{l}{ Heavy metals $(\mathrm{mg} / \mathrm{l})$} \\
\hline & $\mathrm{Fe} / 10$ & $\mathrm{Cu}$ & $\mathrm{Zn}$ & $\mathrm{Pb}$ & $\mathrm{Ni}$ & $\mathrm{Cd}$ \\
ITK17 & 140.6 & 0.049 & 0.007 & 0.0056 & 0.0041 & 0.267 \\
ITK18 & 49.95 & 0.051 & 0.02 & 0.0071 & 0.0536 & 0.312 \\
ITK19 & 171.83 & 0.048 & 0.0058 & 0.0066 & 1.01 & 0.171 \\
ITK20 & 32.35 & 0.065 & 0.031 & 0.019 & 0.0184 & 0.616 \\
ITK21 & 34.57 & 0.181 & 0.029 & 0.283 & 1.075 & 1.096 \\
ITK22 & 35.42 & 0.16 & 0.028 & 0.246 & 1.05 & 1.126 \\
ITK23 & 133.07 & 0.067 & 0.0075 & 0.073 & 0.407 & 0.289 \\
ITK24 & 260.67 & 0.044 & 0.0038 & 0.013 & 0.221 & 0.3871 \\
ITK25 & 69.47 & 0.058 & 0.014 & 0.079 & 0.749 & 0.516 \\
\hline
\end{tabular}

Table 12a. The Contamination factor (CF) of heavy metals in Itakpe dry season sediments

\begin{tabular}{lllllll}
\hline Heavy & \multicolumn{5}{c}{ Sample locations } \\
\hline Metals (mg/l) & ITK08 & ITK09 & ITK11 & ITK12 & ITK13 & ITK15 \\
$\mathrm{Fe}$ & 47.05 & 14.25 & 32.45 & 41.33 & 17.14 & 12.40 \\
$\mathrm{Cu}$ & 5.50 & 0.50 & 35.67 & 36.17 & 1.17 & 0.83 \\
$\mathrm{Zn}$ & 1.12 & 0.48 & 1.28 & 1.55 & 0.49 & 0.38 \\
$\mathrm{~Pb}$ & 1.86 & 0.14 & 3.71 & 6.43 & 0.43 & 0.29 \\
$\mathrm{Ni}$ & 0.64 & 1.11 & 1.35 & 1.95 & 1.16 & 0.35 \\
$\mathrm{Cd}$ & 0.25 & 0.64 & 0.91 & 1.02 & 0.68 & 0.22 \\
\hline
\end{tabular}

Table 12b. Contamination factor (CF) of heavy metal classes (Hakanson, 1980)

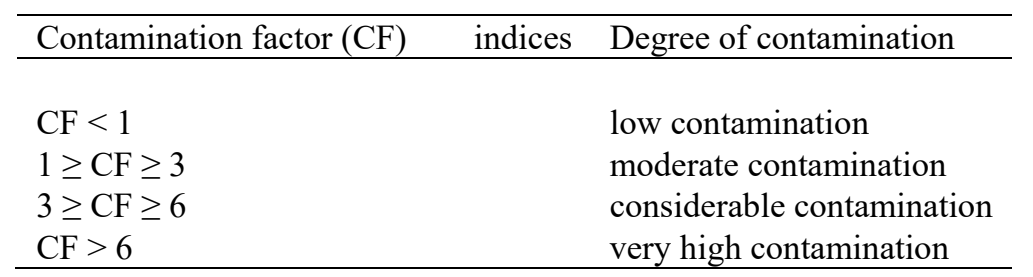


Table 13. The contamination factor (CF) of wet season sediments

\begin{tabular}{lllllll}
\hline Sample location & \multicolumn{7}{l}{ Heavy metals $(\mathrm{mg} / \mathrm{l})$} \\
& $\mathrm{Fe}$ & $\mathrm{Cu}$ & $\mathrm{Zn}$ & $\mathrm{Pb}$ & $\mathrm{Ni}$ & $\mathrm{Cd}$ \\
ITK17 & 80.46 & 3.95 & 0.57 & 0.45 & 0.33 & 21.50 \\
ITK18 & 56.01 & 2.87 & 1.12 & 0.40 & 3.00 & 17.50 \\
ITK19 & 60.59 & 2.90 & 0.35 & 0.40 & 61.33 & 10.33 \\
ITK20 & 18.14 & 1.18 & 0.56 & 0.35 & 0.33 & 11.17 \\
ITK21 & 24.18 & 4.38 & 0.70 & 6.85 & 26.00 & 26.50 \\
ITK22 & 21.91 & 3.51 & 0.62 & 5.40 & 23.00 & 24.67 \\
ITK23 & 62.31 & 4.18 & 0.47 & 4.55 & 25.33 & 18.00 \\
ITK24 & 15.07 & 0.67 & 0.06 & 0.20 & 3.33 & 5.83 \\
ITK25 & 16.47 & 0.95 & 0.24 & 1.30 & 12.33 & 8.50 \\
\hline
\end{tabular}

Tables 14a. The PLI of dry and wet seasons

\begin{tabular}{llllllll}
\hline & & \multicolumn{5}{c}{ Sample locations } \\
\hline Heavy metals (mg/l) & ITK08 & ITK09 & ITK11 & ITK12 & ITK13 & ITK15 & Average PLI \\
PLI & 2.10 & 0.84 & 4.35 & 5.56 & 1.22 & 0.67 & 2.46 \\
\hline
\end{tabular}

\begin{tabular}{lllllllllll}
\hline \multicolumn{10}{c}{ Sample locations } \\
\hline $\begin{array}{l}\text { Heavy } \\
\text { metals } \\
(\mathrm{mg} / 1)\end{array}$ & ITK17 & ITK18 & ITK19 & ITK20 & ITK21 & ITK22 & ITK23 & ITK24 & ITK25 & $\begin{array}{l}\text { Average } \\
\text { PLI } \\
\text { PLI }\end{array}$ \\
2.89 & 3.95 & 4.50 & 1.58 & 8.39 & 7.26 & 7.96 & 1.15 & 2.83 & 4.61 \\
\hline
\end{tabular}

Table 14b. Pollution load index of heavy metal classes (Tomilson et al., 1980)

\begin{tabular}{ll}
\hline PLI indices & Pollution level \\
\hline 0 & Perfection \\
1 & Only baseline levels of pollutants present \\
$>1$ & Progressive deterioration of the site \\
\hline
\end{tabular}

Almost all the locations have experienced site deterioration in both seasons but pollution load intensity is higher in rainy season than dry season (Table 14).

\section{Discussion}

From Table 2, dry season samples have higher mean concentrations (mg/l) than wet season except $\mathrm{Fe}, \mathrm{Cu}$ and $\mathrm{Pb}$ where the reverse is the case (Table 2). The dry season major cations order were $\mathrm{K}>\mathrm{Ca}>\mathrm{Na}>\mathrm{Mg}$ while the heavy metal were $\mathrm{Fe}>\mathrm{Zn}>\mathrm{Cd}>\mathrm{Ni}>\mathrm{Cu}>\mathrm{Pb}$. Major cations order were $\mathrm{K}>\mathrm{Ca}>\mathrm{Na}>\mathrm{Mg}>$ while the heavy metal was $\mathrm{Fe}>\mathrm{Cu}>\mathrm{Cd}>\mathrm{Zn}>\mathrm{Ni}>\mathrm{Pb}$ in wet season.

In the dry season (Table 3), at $\mathrm{P}<0.01$, Ca-Na-Mg-Cu-K-Zn-Fe displayed significant correlations. At $\mathrm{P}<0.05$, $\mathrm{Pb}-\mathrm{Na}-\mathrm{Mg}$ also revealed strong correlations (Table 3). At $\mathrm{P}<0.01, \mathrm{Ca}-\mathrm{K}-\mathrm{Mg}-\mathrm{Fe}-\mathrm{Cu}-\mathrm{Ni}$ and at $\mathrm{P}<0.05$, $\mathrm{Ca}-\mathrm{K}-\mathrm{Mg}-\mathrm{Fe}-\mathrm{Cu}-\mathrm{Ni}$ displayed strong and significant correlations (Table 3). This is an indication that these elements were discharged from same source (Ameh, et al., 2014). The sources of these heavy metals include: iron-ore mining and processing activities, fuel sources, domestic and fertilizers applications and chemical weathering of minerals.

The effects of human activities with respect to all the heavy metals are shown in Table 4. These human sources include: Agricultural lands, fertilizers and biocides application, fuel sources, mining and processing. The anthropogenic factor (\%) for $\mathrm{Fe}, \mathrm{Pb}, \mathrm{Ni}$ and $\mathrm{Cd}$ were higher during the wet season than dry season. The $\mathrm{AF}$ order was $\mathrm{Fe}>\mathrm{Cu}>\mathrm{Pb}>\mathrm{Ni}>\mathrm{Zn}>\mathrm{Cd}$ and $\mathrm{Fe}>\mathrm{Ni}>\mathrm{Cd}>\mathrm{Cu}>\mathrm{Pb}>\mathrm{Zn}$ for dry and rainy seasons respectively. These generally higher concentrations maybe caused by dissolution and subsequent precipitation, adsorption and relative mobility due to runoff (Ameh, et al., 2014).

The Igeo computation showed that $\mathrm{Fe}$ was moderately to highly polluted in dry season while the wet season revealed highly polluted to very highly polluted (Tables 5 and 6). Except in two locations where $\mathrm{Cu}$ was highly 
polluted, other locations recorded background concentrations to moderately unpolluted in both seasons. Zinc in both seasons recorded background concentrations. Lead recorded background concentrations in both seasons but for few locations in wet season where moderately to unpolluted was recorded. While $\mathrm{Ni}$ and $\mathrm{Cd}$ recorded background concentrations during dry season, moderately to highly polluted was observed during wet season. Higher concentrations were generally recorded for $\mathrm{Fe}, \mathrm{Ni}$ and $\mathrm{Cd}$ in wet season than in dry season (Table 5b). The Igeo order was $\mathrm{Fe}>\mathrm{Cu}>\mathrm{Pb}>\mathrm{Ni}>\mathrm{Zn}>\mathrm{Cdand} \mathrm{Fe}>\mathrm{Cd}>\mathrm{Ni}>\mathrm{Pb}>\mathrm{Cu}>\mathrm{Zn}$ respectively for both seasons.

For both seasons, the $\mathrm{E}_{\mathrm{r}}^{\mathrm{i}}$ and RI considered heavy metals ecological impacts on sediments and also associates ecological and environmental effects with toxicity. It evaluates pollution using comparable and equivalent property index grading methods (Table 7).

During the dry season, $\mathrm{Cd}$ and $\mathrm{Pb}$ showed tiny hazard level, $\mathrm{Cu}$ was middle while $\mathrm{Ni}$ and $\mathrm{Zn}$ showed none. Integrated pollution index of 100.1 (tiny) was recorded during dry season. The RI value for wet season of same sediment recorded middle hazard level of $\mathrm{RI}=178$. While $\mathrm{Pb}$ recorded strong hazard level, Ni was middle and $\mathrm{Cu}$ showed tiny hazard level (Tables 7, 8 and 9).

Only Fe showed very high enrichment factor in all locations in dry season (Tables 10 \& 11). Other heavy metals recorded background enrichment factor. All enrichment values in wet season were almost ten times $\left(10^{*}\right)$ the extremely high enrichment value $(\mathrm{EF}>40)$ for Fe. During same season, Ni and Cd revealed depletion to minimal enrichment in few locations while other heavy metals like $\mathrm{Cu}, \mathrm{Zn}$ and $\mathrm{Pb}$ recorded background concentrations $(\mathrm{EF}<1)$. The Dry season EF trend was $\mathrm{Fe}>\mathrm{Cu}>\mathrm{Pb}>\mathrm{Ni}>\mathrm{Zn}>\mathrm{Cd}$ and the wet season trend was $\mathrm{Fe}>\mathrm{Ni}>\mathrm{Cd}>$ $\mathrm{Pb}>\mathrm{Cu}>\mathrm{Zn}$.

The contamination factor for $\mathrm{Fe}$ in both seasons recorded extremely to very high contaminations. Very high contaminations were recorded in some locations with respect to $\mathrm{Cu}$, while low to moderate contaminations were recorded in other locations during dry season. For the same $\mathrm{Cu}$, considerable contaminations were observed during the wet season with few locations showing low to moderate contamination. In wet season, $\mathrm{Zn}$ recorded low contamination in all locations but one $(\mathrm{CF}<1)$ while in dry season, low to moderate contaminations were observed. In wet season also, $\mathrm{Pb}$ recorded low contaminations in most locations except in three other locations where considerable concentrations were revealed. In dry season, low to moderate contaminations were recorded. Nickel and Cadmium revealed low contamination during the dry season. Dry season Cd degree of contamination was low for all locations. Ni revealed low contamination for two locations and moderate contamination for the rest sample points (Tables 12 \&13). In wet season, very high contamination was recorded for both $\mathrm{Ni}$ and $\mathrm{Cd}$. The $\mathrm{CF}$ order for both seasons were $\mathrm{Fe}>\mathrm{Cu}>\mathrm{Pb}>\mathrm{Ni}>\mathrm{Zn}>\mathrm{Cd}$ and $\mathrm{Fe}>\mathrm{Ni}>\mathrm{Cd}>\mathrm{Cu}>\mathrm{Pb}>\mathrm{Zn}$ respectively.

From the five indices, $\mathrm{Fe}, \mathrm{Ni}, \mathrm{Pb}$ (except the $\mathrm{EF}$ ) and $\mathrm{Cd}$ were all higher during rainy season than dry season. Pereira et al., 2008, showed that iron ore mining constitutes potential sources for these heavy metals in sediments. The lower concentrations of $\mathrm{Cu}$ and $\mathrm{Zn}$ during rainy season may be due to lower $\mathrm{pH}$, hardness and sulphide content due largely to mining (Pereira et. al., 2008). Both $\mathrm{Cu}$ and $\mathrm{Zn}$ are very mobile and may have been dispersed by run off and accumulated in the sediments (Eze \& Chukwu, 2011). From similar work carried out by Lin et al., 2012 and Mondol et al., 2011, the high lead concentration in rainy season was attributed to the fact that it was transported in clastic/suspended particles which tend not to enter streams/lakes due to its affinity for sediments; complexation and aggregation with clays, organic matter and chemical fertilizer application. In similar studies by Lin et al., 2012 and Mondol et al., 2011, the higher concentration of lead in rainy season was attributed to high percentage of lead in the air which mixed up with rainwater and finally reached the sediments through precipitation. From this study, cadmium and Ni were also higher in rainy season than dry season. This is in agreement with earlier work by Callender, 2003. According to this author, both heavy metals are associated with soil minerals, they are mobile and may have been carried and discharged/dispersed into the stream sediments by runoff and latter precipitated onto the sediments. Possible reasons for higher Cd could be due to its presence in biocides and fertilizers, gasoline, phosphates and domestic wastes which were easily washed off into the stream sediments during rainy season (Sekabira et al., 2010).

The higher concentration ofNi could also been associated with both amorphous iron and Mn oxides that coated the silica and sand grains (Lin et al., 2012). According to Callender, 2003, another factor responsible for higher $\mathrm{Ni}$ concentration was atmospheric input washed down during rainfall.

The $\mathrm{E}_{\mathrm{i}}^{\mathrm{r}}$ value for $\mathrm{Pb}$ indicated that $\mathrm{Pb}$ level belong to the strong ecological hazard level during rainy season, while $\mathrm{Ni}$ was middle and $\mathrm{Cu}$ tiny levels respectively. The other heavy metals belong to the none hazard (safe) level. The dry season results indicated middle level for $\mathrm{Cu}$ and tiny for $\mathrm{Cd}$ and $\mathrm{Pb}$. Putting the ecological risk 
into perspective, anthropogenic factors have played a major role in $\mathrm{Pb}, \mathrm{Ni}, \mathrm{Cu}$ and $\mathrm{Cd}$ in the sediments in both seasons (Ameh, et al., 2014). While the ecological hazard level (RI) is middle for wet season, it is tiny during dry season in same study area. The enrichment status of the heavy metals $\left(\mathrm{E}_{\mathrm{r}}^{\mathrm{i}}\right)$ showed tiny to middle in dry season while in wet season, it ranged from tiny-middle-strong. This result is in contrast to earlier work by Wenjin and Xinqing, 2013 carried out elsewhere.

Mining, processing and agricultural activities in this area were the major sources of these heavy metals. On the whole, hazard level (RI) in rainy season was at the middle ecological risk level. This calls for frequent monitoring, control and outright clean up of the mining and agricultural activities in this area with particular emphasis on heavy metals such as $\mathrm{Pb}, \mathrm{Ni}, \mathrm{Cu}$ and $\mathrm{Cd}$.

\section{References}

Ameh, E. G., Omatola, O. D., \& Awulu, T. D. (2014). Utilization of environ metric and index methods as water quality assessment tools focusing on heavy metal content of water around Okaba coal mines, Kogi State, Nigeria. Advances in Research, 2(2), 80-94.

Callender, E. (2003). Heavy metals in the environment-Historical trends. In: Environmental Geochemistry, B.S.Lollar (Ed.) vol. 9. Treatise on Geochemistry, H. D. Holland and K. K. Turekian (Eds.). Elsevier-Pergamon, Oxford, 67-107.

Eze, H. N., \& Chukwu, E. (2011). Small scale mining and heavy metal pollution of agricultural soils: A case of Ishiagu mining District, South Eastern Nigeria. Journal of Geology and Mining Research, 3(4), 87-104.

Hakanson, L., (1980). Ecological Risk Index for Aquatic Pollution Control, a Sedimentological Approach. Water Res., 14, 975-1001.

Lin, C., He, M., Liu, S., \& Li, Y. (2012). Contents, enrichment, toxicity and baselines of trace elements in the estuarine and coastal sediments of the Daliao Riversystem, China. Geochemical Journal, 46, 371-380.

Mondol, M. N.,Chamon, A. S., Faiz, B., \& Elahi, S. F. (2011). Seasonal variation of heavy metal concentrationsin water and plant samples around Tejgaon industrial area of Bangladesh. Journal of Bangladesh Academy of Sciences.

Muller, G. (1979). Schwermetalle in den sediments des Rheins-Veranderungen Seitt 1971. Umschan.vol.79: 778-783. In: Chen, C., Kao, C., Chen, C and C. Dong, 2007. Distribution and accumulation of heavy metals in the sediments of Kaohsiung Harbor, Taiwan. Chemosphere, 66, 1431-1440.

Odigi, M. I. (2002). Geochemistry and geotectonic setting of migmatitic gneisses and amphibolites in the Okene-Lokoja area of SW Nigeria. Journal of Mining and Geology, 38(2), 81-89.

Olade, M. A. (1978). General features of a Precambrian iron ore deposit and its environment at Itakpe ridge, Okene, Nigeria. Applied earth science, 87, B1-B10.

Pereira, A. A., Hattum, B. V., Brouwer, A., Bodegom, P. M. V., Rezende, C. E., \& Salomons, W. (2008).Effects ofiron-ore mining and processing on metal bioavailability in a tropical coastal lagoon. J. Soils Sediments, 8 , 239-252.

Qingjie, G., Jun, D., Yunchuan, X., Qingfei, W., \& Liqiang, Y. (2008).Calculating pollution indices by heavy metals in ecological geochemistry assessment and a case study in parks of Beijing. Journal of China University of Geosciences, 19(3), 230-241.

Sekabira, K., Oryem H. O., Basamba, T. A., Mutumba, G., \& Kakudidi, E. (2010). Assessment of heavy metal pollution in the urban stream sediments and its tributaries. International Journal of Environment, Science and Technology, 7(3), 435-446.

Seshan, B. R. R., Natesan, U., \& Deepthi, K. (2010). Geochemical and statistical approach for evaluation of heavy metal pollution in core sediments in southeast coast of India. Int. J. Sci. Tech., 7(2), 291-306.

Sutherland, R. A. (2000). Bed sediment-associated trace metals in an urban stream, Oahu, Hawaii. Environmental Geology, 39, 611-37.

Tomilson, D. C., Wilson, D. J., Harris, C. R., \& Jeffrey, D. W. (1980). Problem in assessment of heavy metals in estuaries and the formation of pollution index. Helgol. Wiss. Meeresunlter, 33(1-4), 566-575.

Wenjin, Y., \& Xinqing, Z. (2013). The distributional characteristics of heavy metals in Jiangsu province shoal sea. Journal of Environmental and Public Health, 2013, 1-8. 


\section{Copyrights}

Copyright for this article is retained by the author(s), with first publication rights granted to the journal.

This is an open-access article distributed under the terms and conditions of the Creative Commons Attribution license (http://creativecommons.org/licenses/by/4.0/). 